\title{
El fraude contable en el Perú: periodo 2009-2016
}

\author{
Ildefonso Rebaza Carpio ${ }^{1}$
}

Recibido: 02 de noviembre de 2019

Aprovado: 10 de diciembre de 2019

Rebaza, I. (2020). El fraude contable en el Perú: periodo 2007-2016. Revista Activos, 18(1), 123-159. DoI: https://doi.org/10.15332/25005278/6161

\section{Clasificación JEL: M41, M42, M48}

\section{Resumen}

En un contexto nacional de escándalos relacionados con la corrupción, esta investigación indaga sobre el fraude contable, más concretamente, sobre el fraude en la emisión de estados financieros en el Perú. El estudio se realizó sobre una muestra conformada por empresas de diversos sectores de actividad que cotizan acciones en la Bolsa de Valores de Lima. El trabajo realizado puso énfasis en la relación funcional entre las variables ventas netas (VN) y el resultado contable antes de impuestos (RCAI). Las evidencias demostraron que, durante el periodo examinado (20072016), el fraude se constituyó en una práctica habitual y extendida en

1 Contador Público Colegiado, magíster en Contabilidad y magíster en Ciencias. Correo electrónico irebazac@gmail.com.

ORCID: https://orcid.org/0000-0003-4933-7301 
nuestro país. En esa línea, la nivelación de resultados fue el instrumento privilegiado.

Palabras clave: fraude, fraude contable, conducta, racionalidad.

\title{
Accounting Fraud in Peru: 2009-2016
}

\begin{abstract}
In a national context of corruption-related scandals, this research scrutinizes accounting fraud and, more specifically, fraud in the issuance of financial statements in Peru. The study was carried out on a sample made up of companies from various activities sectors listed on the Lima Stock Exchange. The research emphasized the functional relationship between net sales variables and pre-tax accounting results. Evidence showed that, during the period examined (2007-2016), fraud was a common and widespread practice in our country. In that vein, the levelling of results was the privileged instrument.
\end{abstract}

Keywords: fraud, accounting fraud, conduct, rationality. 


\section{Introducción}

Entre los primeros objetivos de la contabilidad se encuentra el de producir información financiera veraz, objetiva y confiable para los usuarios. En las últimas décadas, sin embargo, dichos fines parecen haber sido soslayados por la práctica profesional: el mundo ha sido sacudido por el develamiento de numerosos fraudes (Enron y WorldCom, en Estados Unidos de Norteamérica; Parmalat, en Italia; Olympus, en Japón; entre otros) en los que la contabilidad se ha visto involucrada como instrumento de acción. En dicho escenario, conforme a todas las evidencias, el procedimiento preferido ha sido alterar la información contable; una rutina que no parece ser extraña en nuestro medio profesional.

Efectivamente, en torno a este tema, un informe de investigación de Convoca, en Perú, sugiere que "abogados, auditores y empresarios" se confabulan para eludir el pago de impuestos (Gutiérrez, 2017). De otro lado, en los sucesos conocidos como Lava Jato, firmas constructoras brasileñas, consorciadas con empresas peruanas, "obtenían fondos para [el pago de] sobornos [surgidos] de sobrecostos de obras [..., que no eran declarados contablemente" ("Odebrecht...", 2017). También, en el año 2015, un alto representante de la Confederación Nacional de Instituciones empresariales (Confiep) y, al mismo tiempo, presidente de la Cámara Peruana de la Construcción (Capeco), fue condenado (por defraudación tributaria) por la emisión y registro de facturas falsas (Carreño, 2017). En fin, la Superintendencia Nacional de Administración Tributaria y Aduanas (Sunat) reveló que el incumplimiento de pago del impuesto general a las ventas (IGV), en todas las modalidades de evasión, alcanza la suma de S/ 23000 millones anuales, siendo la omisión de ventas la modalidad más empleada (“Se evaden..., 2018).

Los eventos aludidos dan cuenta de una práctica que no respeta fronteras. En nuestro medio, sin embargo, el fenómeno no parece haber llamado la atención de los investigadores contables. En esa línea de pensamiento nos preguntamos acerca del alcance del fenómeno del fraude contable en el Perú. 
Como acción incorrecta cometida en perjuicio de terceros, el fraude es una manifestación de la conducta humana. Este puede ser definido como "una falsa representación de la verdad o el ocultamiento de un hecho material para inducir a otros a actuar en detrimento de sí mismos" (Association of Certified Fraud Examiners [ACFE], 2012). En el dominio contable, el fenómeno se estudia desde dos ópticas: (i) el fraude corporativo², y (ii) el fraude de los estados financieros (Bayou y Reinstein, 2001). En el primer caso, se trata de la malversación clara y deliberada o del empleo incorrecto de los recursos (activos, derechos, información, etc.) de una organización. En el segundo caso, el objetivo es generar estados financieros fraudulentos; es decir, producir información sesgada, distante de la realidad. Este último es el tema que concentrará nuestros esfuerzos.

En el escenario expuesto, este estudio pretende determinar si, desde la información financiera que producen las empresas peruanas, se encuentran libres de desviaciones y alteraciones. Para tales fines se pondrá énfasis en las cifras del estado de ganancias y pérdidas. Así, (a) se examinará el comportamiento de dos variables: ventas netas y resultado contable antes de impuestos; (b) se estudiará la influencia de las variables mediadoras o intervinientes costo de ventas, gastos operativos y otros ingresos o gastos en la relación entre las variables, y (b) se aludirá a las posibles causas subyacentes en dichas conductas.

El esfuerzo a emprender se justifica por la importancia del rol social de la contabilidad, la misma que se sostiene en premisas como las siguientes: (a) los informes contables son documentos indispensables para la recaudación de diversos e importantes tributos; (b) la estructura financiera presentada por la contabilidad es determinante para acceder a créditos e inversiones; (c) los resultados contables son empleados para solucionar demandas salariales; (d) la proporcionalidad de la propiedad del patrimonio

2 En la web es posible encontrar el estudio de Ernst \& Young: "Construyendo un ambiente ético. Estudio sobre el riesgo de fraude contable en el Perú. Enero 2014”. Sobre el mismo tema, KPMG ha publicado "El fraude corporativo en Latinoamérica 2008-2010”. 
contable sirve de base para distribución de utilidades, entre otros. En ese contexto, resulta claro que toda alteración de cifras atenta contra los intereses de los agentes involucrados (Estado, trabajadores, acreedores, accionistas). De otro lado, en la medida en que, al parecer, se trata de un tema no abordado en nuestro medio profesional, su estudio aportará, adicionalmente, metodología, teorías y técnicas para estudios futuros en el dominio de las ciencias empresariales.

Para el desarrollo del presente trabajo, la fuente principal de información ha sido la Superintendencia del Mercado de Valores del Perú. Ella, de ordinario, pone a disposición de los interesados los estados financieros individuales auditados de (en promedio) 282 empresas que cotizan acciones en la Bolsa de Valores de Lima. De sus registros, seleccionamos inicialmente una muestra conformada por 30 empresas (10.6\% del universo) relevantes en sus sectores de actividad con la finalidad de examinar el periodo 2007-2016, una etapa de crecimiento de la economía peruana. En torno a este tema, debido a factores vinculados con (a) cese de actividades, (b) discordancia (en el tiempo) de la información, y (c) la exclusión de algunas empresas (inicialmente seleccionadas) de los registros de la Bolsa de Valores, la muestra se vio reducida a 19 observaciones (6.7 \% del universo).

El texto que sigue a continuación se estructura de la siguiente manera: (a) marco teórico, refiere a los modelos teóricos propuestos, y a las teorías susceptibles de ayudar a explicar los resultados que se esperan; (b) metodología, trata sobre la formulación y operacionalización de la hipótesis; analiza y discute los resultados; (c) conclusiones y perspectivas futuras, resume en pocas palabras, los resultados del examen de la hipótesis examinada. Propone alternativas para estudios futuros vinculados con el trabajo desarrollado. 


\section{Marco teórico}

Los estados financieros son el resultado de dos procesos ${ }^{3}$ : (a) político, y (b) contractual. El primero alude a un conjunto de pasos interrelacionados, determinados por la forma en que se asigna la riqueza en una sociedad (p. ej., el destino de la recaudación de los impuestos). En dicho escenario, en la medida en que en el proceso político participan grupos con intereses opuestos y que el poder político se ejerce desde el Estado, la idea de confrontación se manifiesta como fenómeno latente. El proceso contractual, en segundo lugar, apunta a (a) las motivaciones que subyacen en la (s) elección de métodos y procedimientos contables, y a (b) la predicción del comportamiento de los agentes en el proceso de producción de los estados financieros. Bajo tales premisas, si es la información contable un instrumento redistributivo, toda alteración de sus cifras tenderá a agudizar los antagonismos, tanto internos (p. ej., entre accionistas, ejecutivos y trabajadores) como externos (p. ej., inversionistas, acreedores, el Estado) (Villarroya, 2003, p. 37).

En el marco expuesto, toda distorsión de las cifras contables es el resultado de una "conducta intencionada - por acción u omisión - que culmina en la distorsión de estados financieros". Ella se exterioriza mediante (a) la manipulación de cifras, (b) la falsificación de documentos y alteración de registros, o bien (c) produciendo estados financieros erróneos, u omitiendo cifras, obligaciones o cualquier otra información. En esa línea, la intención tiene como propósito engañar, perjudicar o distorsionar la información financiera (García Benau y Humprey, 1995, p. 700). En concordancia con lo anterior, el American Institute of Certified Public Accountants (AIPAC, 2002) ha definido al fraude contable como "un acto intencional que resulta en un error material de los estados financieros". Desde dicha perspectiva, en primer lugar, diversos modelos teóricos han sido propuestos para estudiar

3 Un proceso puede definirse como el "conjunto de operaciones lógicas y aritméticas ordenadas con la finalidad de obtener un resultado determinado" (“Proceso", 2005). 
el tema en cuestión y, en segundo lugar, se han planteado diversas teorías explicativas.

\section{Modelos teóricos}

En general, tres perspectivas han sido propuestos para estudiar el fraude: (a) el triángulo del fraude, (b) el diamante del fraude y (c) el fraude como sistema. Desde nuestra óptica, los tres modelos contribuyen a comprender mejor el fenómeno en estudio. En esa línea, buscaremos complementar los atributos de cada perspectiva con la finalidad de construir una sólida fundamentación teórica.

\section{El triángulo del fraude}

De acuerdo con Abdullai y Mansor (2015, p. 38), la propuesta tiene sus orígenes en el trabajo de Edwin Sutherland (1939). Posteriormente, según los mismos autores, Cressy (1950) —uno de los discípulos de Sutherland (1939) - se concentró en identificar los elementos necesarios para la ocurrencia de un fraude, con lo que sugirió los siguientes: (a) los motivos -o presiones - del infractor, (b) la racionalidad del transgresor - la evaluación de las probabilidades de ser detectado - y (c) la oportunidad para cometer el acto fraudulento. Para este punto de vista, las oportunidades para defraudar se presentan en contextos vulnerables, en escenarios potencialmente atractivos, y con aparente poco riesgo para ser detectados (o penalizados). En situaciones como las expuestas, se sostiene que la acción fraudulenta es una elección (Ramamoorti, 2008, pp. 522-525).

Un reciente estudio realizado por Ajekwe e Ibiamke (2017) analizó críticamente la literatura relacionada con la motivación y con los escenarios de la producción de la información financiera fraudulenta. El trabajo tuvo como objetivo mejorar la comprensión del fraude con la finalidad de optimizar la capacidad para detectarlo. Entre sus conclusiones, los autores sostienen que (a) gran parte de las investigaciones precedentes priorizaron el estudio de los factores motivacionales y circunstanciales del fraude a 
nivel social, organizacional e individual, y que (b) en la medida en que la mayor parte de las investigaciones desarrolladas reposaron en evidencias obtenidas en Estados Unidos de Norteamérica, ellas podrían no ser replicables en otros países.

Desde la perspectiva mencionada, el trabajo de Villarroya (2003, p. 23) se planteó entre sus objetivos principales la identificación de causas probables de conductas fraudulentas. En un contexto español, con una muestra de 110 empresas, los resultados de su estudio proponen que la conducta fraudulenta está delimitada por tres factores: (a) la predisposición: "La ausencia de ética es el motor de la conducta fraudulenta y el soslayo de lo moral es el mecanismo inhibidor del sentimiento de culpa"; (b) la oportunidad: "Los mecanismos de control y el sistema de incentivos juegan un importante rol en la comisión de la conducta fraudulenta”; y (c) la decisión: determinada por la valoración que realiza el infractor con respecto al beneficio esperado y a los riesgos asumidos (Villarroya, 2003, p. 315).

Finalmente, un estudio de Abdullahi y Mansor (2015) concluyó en que los fraudes están motivados por el riesgo percibido y por las tentaciones de obtener un beneficio, factores que conforman la teoría del triángulo del fraude de Cressy (1950).

\section{El diamante del fraude}

Según Abdullai y Mansor (2015, pp. 42-43), Wolfe y Hermanson (2004) propusieron el "diamante del fraude" como una extensión, o una versión mejorada, del triángulo del fraude. A tal fin adicionaron, a los elementos ya conocidos, el de la "capacidad" del transgresor, entendiendo lo siguiente:

1. La posición que ocupa o la función que desempeña el infractor con respecto al potencial agraviado, en el entendido que ella (la posición o función) puede facilitar la acción transgresora.

2. La inteligencia, experiencia y creatividad del transgresor. Personas con tales cualidades, susceptibles de comprender las debilidades de 
control, son las más propensas a cometer los fraudes materialmente más importantes.

3. El ego del agresor. Generalmente, el defraudador confía en que no será detectado o cree que, de ser atrapado, saldrá fácilmente del problema. Una conducta de esta naturaleza afecta su análisis acerca del costobeneficio de la potencial acción fraudulenta.

4. Otras características. El infractor debe tener capacidad para (i) coaccionar a otros para participar en un fraude o para ocultarlo; (ii) atemorizar, para plantear exigencias inusuales y significativas; (iii) mentir con eficacia y consistencia, y (iv) saber administrar situaciones estresantes.

Para Wolfe y Hermanson (2004, citados por Abdullai y Mansor, 2015, p. 43), sin las cualidades expuestas, el infractor no podría perpetrar -ni ocultar-con éxito la acción fraudulenta.

\section{El fraude como sistema}

Para Bayou y Reinstein (2001, pp. 387-394), en la medida en que (a) se repite, (b) evoluciona a lo largo de los años, (c) adopta estrategias de simulación (para permanecer oculto) y de (d) disminución (con la finalidad de no dejar rastro), el fraude contable acontece al interior de un sistema. Este se conforma con los siguientes componentes:

1. Las razones. Aluden a los elementos que conducen al fraude: la motivación y la oportunidad. La primera hace referencia a los incentivos o motivos para cometer la infracción. En contabilidad, por ejemplo, los motivos pueden ser: (a) producir información distorsionada o (b) desviar activos. La segunda alude al convencimiento (del infractor) de que existe una "circunstancia favorable para solucionar un problema personal de manera clandestina, violando la confianza”. Ambos conceptos se influencian mutuamente. En ese sentido, "a mayor motivación mayor urgencia para detectar debilidades de control para acelerar el proceso del fraude" (p. 390). 
2. La intención. Mientras que el defraudador es un individuo que tiene la intención de defraudar ("el problema de la intención"), la defraudación es el objetivo de dicha intención (el evento). En el contexto de un error intencional, el objetivo puede adoptar la forma cualitativa, cuantitativa y ser aleatorio. En un error no intencional, puede tomar la forma cuantitativa y aleatoria. Mientras el primero busca engañar, el segundo es, a menudo, un problema técnico.

3. El esquema. Los fraudes responden a esquemas. Estos (i) son bocetos detallados exponiendo la forma de llevar a cabo la acción, y (ii) son productos de estados mentales.

Ahora bien, en la medida en que la motivación forma parte del estado mental, es posible aplicar el razonamiento al plan del fraude y a sus relaciones con los otros componentes. Los esquemas de fraude cuentan con una característica de repetitividad: su empleo puede continuar a través de los años, aunque tengan necesidad de ajustes en su referencia a propiedades concretas: por ejemplo, individuos, activos, situaciones, fechas

4. La acción. La acción del fraude difiere del objeto del fraude. La primera alude a un acto, una actividad, una tarea, una maniobra. La segunda refiere al objetivo de la actividad. Los fraudes en estados financieros acontecen por acción u omisión. Por ejemplo, (i) manipulación, falsificación o alteración de registros o documentos de sustento, (ii) tergiversación u omisión de eventos, transacciones u otras informaciones importantes, o (iii) aplicación errónea de las normas contables.

5. El objetivo. El fraude puede tener diferentes objetivos, según el interés del infractor. El fin último del fraude, sin embargo, no es al objeto de la defraudación; es la repetición de la acción y del ciclo. Para ello, requiere de la clandestinidad. En este extremo, el fraude se convierte en una actividad interminable. 


\section{Algunas teorías explicativas}

De acuerdo con Watts y Zimmerman (1986), para comprender los fenómenos contables es menester estudiar la conducta de los agentes en el proceso de generación de información financiera. En ese contexto, siendo el fraude contable una expresión de la acción humana, es necesario recurrir a supuestos de disciplinas afines (p. ej., psicológicas, económicas, filosóficas, etc.) para comprender mejor las motivaciones de los agentes involucrados y tratar de explicar las posibles causas de dichos comportamientos. En esa línea, consideramos pertinente apoyarnos en las teorías que se esbozan a continuación con la finalidad de sostener nuestros puntos de vista con respecto al fraude contable en el Perú.

\section{La conducta humana}

La conducta está determinada por factores que provienen de dos fuentes: (a) genéticas o (b) situacionales. Las primeras están vinculadas con la conducta innata: estas nos acompañan cuando llegamos al mundo. Las segundas aluden a la conducta aprendida, estas se manifiestan ante una situación determinada. Si bien la mayor parte de nuestras conductas son aprendidas, ambas (genéticas y situacionales) son invariables. Esto significa que, dados estímulos similares, las conductas (respuestas a estímulos) siempre serán las mismas (Carthy, 1969, p 23). Bajo tales premisas, "la conducta puede ser definida como una reacción (observable) que un organismo equipado con un sistema nervioso ejecuta como respuesta a estímulos del medio que lo rodea" (Robert, 1984, p. 170). Bajo las premisas expuestas, en un escenario contable, es posible, entonces: (a) pronosticar la conducta cuando se conoce el estímulo (es decir, la motivación), y (b) conocer la naturaleza del estímulo cuando se observa la reacción (es decir, la conducta).

\section{La motivación}

En términos simples, la motivación puede ser definida como "el conjunto de factores internos o externos que determinan, en parte, las acciones de 
una persona" (Real Academia de la Lengua Española, 2019). En vocablos más precisos, a la motivación se le puede conceptuar como un fenómeno psicológico; es decir, como "un conjunto concatenado de procesos psíquicos, que despierta, inicia, mantiene, fortalece o debilita la intensidad del comportamiento, y pone fin al mismo una vez lograda la meta perseguida" (González, 2008, p. 52).

Las principales corrientes de pensamiento sobre la motivación son (a) la conductual, que sostiene que las personas actúan motivadas por los beneficios que puedan obtener; (b) la humanista, que pone énfasis en las cualidades del ser humano, en su capacidad para desarrollar y en su libertad para actuar, y (c) la cognitiva, que considera que las percepciones de los eventos influyen sobre las emociones y los comportamientos. En este estudio, el énfasis es puesto en los argumentos de la corriente conductual.

Desde la perspectiva conductual se sostiene que, en primer lugar, las gratificaciones y las sanciones son centrales en la motivación de las personas (Santrock, 2002; citado por Naranjo, 2009) y, en segundo lugar, que las personas se comportan en atención a las recompensas a conseguir. En esa línea, una conducta es factible de ser modificada si se emplean métodos como los siguientes (Naranjo, 2009, pp. 156-157):

1. Los refuerzos. Pueden ser positivos o negativos. Los primeros se emplean para acrecentar la probabilidad de que una respuesta esperada ocurra. Al refuerzo negativo se le conoce también como estímulo aversivo y se emplea, al igual que el reforzamiento positivo, para incrementar o conservar la posibilidad de una conducta.

2. La extinción. Consiste en dejar de reforzar una conducta: esta tiende a la extinción cuando no se presenta el estímulo reforzador que la sustenta. En otras palabras, al no reforzar un comportamiento este se va debilitando, hasta disminuir la probabilidad de su repetición. 
3. El castigo. Con este método se administra una consecuencia aversiva a la aparición de una conducta. Aquí se trata de que la persona aprenda lo que no tiene que hacer.

\section{La teoría de la elección racional}

La teoría de la elección racional es una perspectiva general del comportamiento humano. En la medida en que su campo de acción es el de la interacción humana, ella es aplicable a escenarios sociales (Vidal de la Rosa, 2008, p. 222). De acuerdo con esta teoría, en toda sociedad el comportamiento de las personas no es arbitrario. Tampoco está motivado por los sentimientos. Las personas tienden, más bien, a desarrollar estrategias de maximización de sus intereses en función a los recursos con que cuentan (Paramio, 2000, p. 66). En otras palabras, las premisas de la teoría de la elección racional respecto del hombre se resumen a las siguientes: (a) el hombre es egoísta por naturaleza y busca (racionalmente) satisfacer su propio beneficio; (b) solo los actos individuales son verificables (empirismo individualista); y (c) solo existe el individuo (ontología individualista) (González, 1994, citado por González Cruz, 2012, pp. 6-7).

\section{La racionalidad instrumental de la contabilidad}

Para darle sentido a los fenómenos sociales, Weber incorporó la noción de racionalidad para comprender la acción social. Para tales fines, la definió como la conducta intencionada de un actor particular dentro de un proceso de desarrollo relacionado con la conducta de otros. Así, en la medida en que los seres humanos aprobamos las acciones sociales, la conducta forma parte de un fenómeno colectivo. En ese escenario, la acción es social solo cuando está orientada por las acciones de otros (Rodríguez, 2014).

Para la racionalidad instrumental, lo racional es lo útil. Su preocupación es la elección de los medios adecuados. En dicho escenario, los objetos son vistos teniendo en cuenta su función relacionada con el logro de un fin específico y no como un fin en sí mismo. En otras palabras, bajo la racionalidad 
instrumental, se dosifican los medios para alcanzar los fines establecidos. Se trata, entonces, de controlar el comportamiento y las acciones en función a un fin señalado como provechoso. En este proceso, los medios son apreciados y definidos por una relación costo-beneficio.

En atención a lo arriba expuesto, en la sociedad capitalista moderna la racionalidad contable tiene dos fines fundamentales: la estimación de los beneficios y la utilidad de la información para la toma de decisiones. Mientras que la última está determinada por el contexto y las circunstancias específicas en las cuales se aplica la racionalidad, la primera es la síntesis del procesamiento de los datos pero también la manifestación de la racionalidad instrumental capitalista. En ese contexto, sin embargo, el beneficio no se puede determinar en términos de satisfacción o bienestar, y debido a que la satisfacción y el bienestar son subjetivos, el beneficio no se puede medir en términos de valor (Giraldo, 2007, pp. 140-150).

\section{La teoría contable positiva}

La teoría contable positiva se interesa en la explicación y predicción de la práctica contable. En atención a tales fines, ella trata de proporcionar argumentos para justificar la acción (la conducta) contable, así como para predecir la materialización de fenómenos contables. Es positiva porque reposa en los "hechos" (lo que "es"), en oposición a prescripciones normativas (lo que "debe ser") (Whitley, 1988, p. 632).

Los estudios vinculados a la teoría contable positiva comprenden dos corrientes: (a) "la investigación sobre el contenido informacional de las cifras contables, y (b) la investigación sobre las motivaciones de la selección contable (p. ej., métodos, procedimientos, prácticas)" (Tremblay et ál., 1994, p. 119). El presente estudio está relacionado con la segunda perspectiva. De acuerdo con ella, "no es posible explicar la práctica contable sin estudiar la racionalidad económica" de los agentes que producen los estados financieros (Tremblay et ál., 1994, p. 121). 


\section{La teoría de la información}

La información puede ser definida como un conjunto de datos organizados y procesados para construir mensajes que, transmitidos por un emisor, modifican el estado de conocimientos del receptor (Feltham, 1968). En la medida en que la contabilidad realiza dichas funciones, ella puede ser percibida como instrumento productor (emisor) de información financiera ${ }^{4}$. En torno a este tema, la literatura especializada propone que todo proceso de producción de información genera ventajas para el emisor: por ejemplo, la posibilidad de (a) alcanzar beneficios, (b) dirigir la percepción de los usuarios, o (c) sesgar la información. En esa línea de pensamiento se acepta que, ante la eventualidad de una transacción, el emisor cuenta con más información y de mejor calidad que la que posee el usuario ${ }^{5}$, circunstancia que, evidentemente, favorece al primero.

En la línea expuesta, la contabilidad es vista como un instrumento para producir información. Como tal, la utilidad de su empleo está en función de los objetivos de quien la utiliza. A este respecto, de acuerdo con el supuesto de maximización económica, "al tomar una decisión, los individuos buscan de maximizar su propio beneficio” (Tremblay et ál., 1994, p. 122), no debería sorprender que, al revelar información, los dirigentes empresariales (a) utilicen la asimetría para sesgar información, (b) empleen sus reportes para manipular percepciones y decisiones de los usuarios, o (c) manipulen contenidos y falseen documentos, con el fin de dirigir los juicios sobre performances y perspectivas futuras. En términos más directos, según la literatura especializada, los emisores emplean su "discreción" para alterar la información financiera y desorientar a los usuarios (Merkl-Davies y Brennan, 2007, p. 181).

\footnotetext{
4 Marco Conceptual de la Información Financiera 2010: el principal objetivo de la contabilidad es el de producir información financiera.

5 Tal situación se explica por la "asimetría de la información”. Esta refiere el hecho de que, en una transacción, uno de los intervinientes puede estar mejor informado que su contraparte. En tal escenario es de suponer que aquel mejor informado tomará mejores decisiones, en detrimento del menos informado quien, probablemente, sufrirá pérdidas (Las Finanzas, s.f.).
} 


\section{Formulación y operacionalización de la hipótesis}

\section{La hipótesis}

Objeto de estudio en este trabajo es el fenómeno del fraude contable. En esa línea, nuestro interés se concentra en indagar acerca del uso, habitualidad y alcance de su práctica en el Perú. Para tales fines, se plantea la siguiente proposición:

$\mathbf{H}_{\mathbf{1}}$ : El fraude contable constituye una práctica habitual en el Perú.

El propuesto sugiere que el fraude en los estados financieros constituye una práctica cotidiana en nuestro medio profesional. Los indicios referidos en la introducción parecen confirmarlo. A pesar de ello, no se conocen estudios desarrollados con muestras de empresas nacionales publicados en nuestro país. Se trataría entonces de un primer esfuerzo para profundizar en el tema.

\section{Las variables}

La hipótesis alternativa $\left(\mathrm{H}_{1}\right)$ será verificada examinando el comportamiento de dos de las principales variables que forman parte del estado de resultados de las empresas que conforman la muestra. Una de ellas, la cuenta de "ventas netas", representa la variable independiente. La otra, la partida "resultado contable antes de impuestos", representa a la variable dependiente. La hipótesis se sustenta en el hecho de que, desde una perspectiva contable, el resultado contable es una función de las ventas netas. De allí se infiere que, teóricamente, cada unidad de crecimiento de estas últimas debería ser seguida por un crecimiento marginal de la primera. Así expuesta, la relación sugiere que mayores ventas deberían generar mayores resultados contables. La no ocurrencia de lo descrito consentiría suponer la alteración de las cifras. En esa línea se manifiestan Cormier, 
Magnan y Morard (1998 p. 26) ${ }^{6}$ cuando sostienen que las empresas tienden a minimizar la volatilidad de sus resultados.

En el escenario expuesto, las partidas restantes, conformantes del estado contable aludido serán agrupadas bajo la etiqueta de "variables moderadoras o intervinientes". Estas son: "costo de ventas"; "gastos operativos"; "otros ingresos/gastos". Bajo tales premisas, la ecuación que expone la relación funcional de las partidas que conforman el estado de resultados puede expresarse en los términos siguiente:

$\mathrm{RCAI}=\mathrm{VN}-(\mathrm{CV}+\mathrm{GO}+/-\mathrm{OT})$

donde:

$\mathrm{RCAI}=$ resultado contable antes de impuesto a la renta.

$\mathrm{vN}=$ ventas netas (o ingresos netos).

$\mathrm{CV}=$ costo de ventas de los bienes transferidos o de los servicios prestados.

GO = gastos de administración y de ventas necesarios en la gestión.

от $=$ otros ingresos/gastos incurridos en el desarrollo de actividades.

La figura siguiente muestra la forma en que las variables moderadoras atemperan la relación entre las variables a estudiar:

$6 \quad$ Sobre la materia, pueden revisarse los trabajos de Sylvie Chalayer (1995) y Breton y Chenail (1997), entre otros. 
Figura 1. Relación entre variables

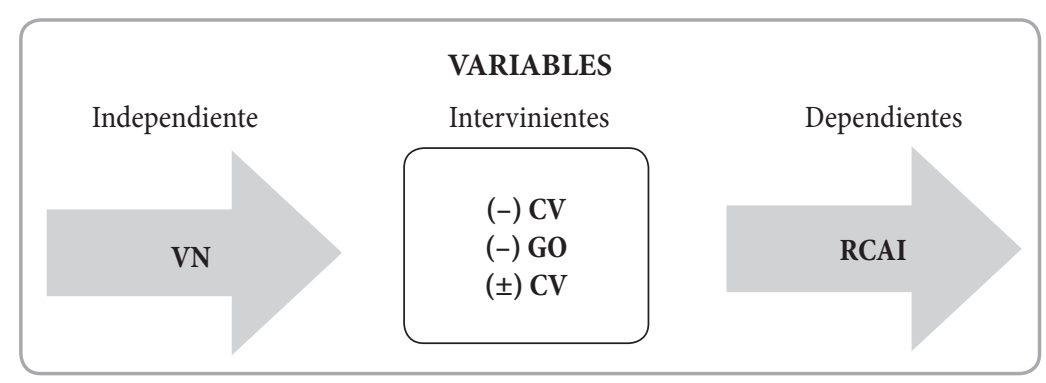

Fuente: elaboración propia.

\section{Aspectos metodológicos}

El desarrollo de un estudio como el propuesto requiere (a) describir las características de un fenómeno particular, (b) identificar las causas posibles de su expresión, y (c) predecir la conducta de una variable dependiente a partir de otra independiente. Tales características sugieren la necesidad de emplear tres perspectivas de estudio: descriptivo, relacional y explicativo. En ese contexto, en la medida en que las observaciones son numéricas, el enfoque privilegiado es el cuantitativo.

Desde otra óptica, todos los métodos de investigación tienen limitaciones. Bajo tal premisa, consideramos que el empleo diversificado de la observación, la inducción, la deducción, del análisis y de la síntesis fortalecerán las inferencias deducibles de la información proveniente de la muestra.

Más concretamente, el objetivo de este trabajo es estudiar el fraude contable en el Perú. Más concretamente, se trata de demostrar que en nuestro país se práctica el fraude en los estados financieros. Para tales fines emplearemos los instrumentos de análisis siguientes:

a. Diagrama de tendencia de las variables de estudio y de las variables moderadoras 
b. Coeficiente de correlación

c. Regresión lineal múltiple

Para los fines expuestos, el modelo de ecuación será el siguiente:

$$
\hat{\mathrm{Y}}=a+b^{1} X^{1}+b^{2} X^{2} \ldots
$$

donde:

$\hat{Y}=$ valor de la variable dependiente

$\mathrm{a}=$ intersección con $\mathrm{Y}$

$\mathrm{X}_{1,2}$, = valores de la variable independiente

$\mathrm{b}_{1,2},=$ pendientes asociadas con las variables independientes

\section{Análisis y discusión de resultados}

La muestra de este estudio está conformada por diecinueve (19) empresas relevantes en sus sectores de actividad. Su composición es diversificada, en los términos que se muestran en en la tabla 1.

Tabla 1. Composición de la muestra

\begin{tabular}{|l|c|}
\hline \multicolumn{1}{|c|}{ Sectores de actividad } & Cantidad de empresas \\
\hline Eléctricas & 7 \\
\hline Agroindustrias & 4 \\
\hline Cerveceras & 3 \\
\hline Otros sectores diversos & 5 \\
\hline Total & $\mathbf{1 9}$ \\
\hline
\end{tabular}

Fuente: elaboración propia. 
Una primera exigencia vinculada con la validez interna del estudio demanda referirse a la homogeneidad de las cifras a examinar. En esa línea, la tabla 2 expone las estadísticas descriptivas de las variables involucradas: una medida de tendencia central (la media) y una de dispersión (la desviación estándar). A este respecto, las notaciones parecen señalar una razonable agrupación de datos alrededor de la media, eventualidad que asegura la comparabilidad de los valores.

Tabla 2. Homogeneidad de los valores

\begin{tabular}{|l|c|c|}
\hline \multicolumn{1}{|c|}{ Variables } & Media & Desviación \\
\hline Ventas netas & 962.30 & 202.47 \\
\hline Costo de ventas & 590.40 & 126.52 \\
\hline Gastos operativos & 157.70 & 32.66 \\
\hline Otros ingresos/gastos & 12.10 & 12.41 \\
\hline Resultado contable antes de impuestos & 202.40 & 43.07 \\
\hline
\end{tabular}

Fuente: elaboración propia.

Una segunda exigencia tiene que ver con la validez del modelo de regresión propuesto. En esa línea, la tabla 3 expone el índice de correlación de Pearson entre las variables implicadas en la investigación. En todos los casos, dicho indicador es positivo. En cuanto a las variables principales se refiere, el indicador es contundente: 0.920 de correlación. De otro lado, los índices de significación inferiores a $p \leq 0.005$ rechazan la hipótesis de no linealidad. 
Tabla 3. Índices de correlación

\begin{tabular}{|l|c|c|c|c|c|}
\hline Variables & $\mathbf{V N}$ & $\mathbf{C V}$ & $\mathbf{G O}$ & $\mathbf{O T}$ & RCAI \\
\hline \multirow{2}{*}{$\begin{array}{l}\text { Ventas netas } \\
\text { Sig. }\end{array}$} & 1 & 0.994 & 0.980 & 0.390 & 0.920 \\
\cline { 2 - 6 } Costo de ventas & & 0.000 & 0.000 & 0.132 & 0.000 \\
\cline { 2 - 6 } Sig. & 0.994 & 1 & 0.973 & 0.368 & 0.882 \\
\hline \multirow{2}{*}{$\begin{array}{l}\text { Gastos operativos } \\
\text { Sig. }\end{array}$} & 0.980 & 0.973 & 1 & 0.291 & 0.905 \\
\cline { 2 - 6 } & 0.000 & 0.000 & 0.000 & 0.147 & 0.000 \\
\hline \multirow{2}{*}{$\begin{array}{l}\text { Otros } \\
\text { Sig. }\end{array}$} & 0.390 & 0.368 & 0.291 & 1 & 0.234 \\
\cline { 2 - 6 } & 0.132 & 0.147 & 0.207 & & 0.258 \\
\hline $\begin{array}{l}\text { Resultado contable antes } \\
\text { de impuestos }\end{array}$ & 0.920 & 0.882 & 0.905 & 0.234 & 1 \\
\cline { 2 - 6 } Sig. & 0.000 & 0.000 & 0.000 & 0.258 & \\
\hline
\end{tabular}

Fuente: elaboración propia.

A primera vista, puede llamar la atención el nivel de significancia (0.258) entre las variables resultados contable antes de impuestos y otros ingresos/ gastos. Tal particularidad, sin embargo, parece superarse por otro importante indicador: el coeficiente de determinación $\left(\mathrm{R}^{2}\right)$. Este informa acerca de la fortaleza de la relación entre las variables. En el presente caso, en su conjunto, las variables predictoras independiente y moderadoras explican 0.998 \% de la variación de la variable dependiente. En esa línea, el error estándar - un indicador de la precisión de la predicción — confirma que el modelo de regresión propuesto es mejor predictor que la media aritmética. Así, en tanto se ubica $\mathrm{R}^{2}$ cerca de 1 , puede afirmarse que las observaciones se ubican alrededor de la línea de regresión (tabla 4). 
Tabla 4. El error estándar

\begin{tabular}{|l|c|c|}
\hline \multicolumn{1}{|c|}{ Variables } & $\mathbf{R}^{\mathbf{2}}$ & Error estándar \\
\hline $\begin{array}{l}\text { Predictora y moderadoras } \\
\text { Dependiente: resultado contable antes de impuestos }\end{array}$ & 0.998 & 2,534 \\
\hline
\end{tabular}

Fuente: elaboración propia.

Desde otra óptica, el coeficiente "F" examina la presunción de que la pendiente de la ecuación es cero. En ese contexto, un valor grande de F es indicativo de que la variable independiente explica mejor la variación de la variable dependiente. Bajo las premisas expuestas, en la medida en que $\mathrm{p} \leq 0.0005$, la $\mathrm{H}_{0}$ es rechazada. Esto significa que el plano definido por la ecuación de la regresión ofrece un buen ajuste de la nube de puntos (tabla 5).

Tabla 5. El coeficiente F

\begin{tabular}{|c|c|c|}
\hline Variables & $\mathbf{R}^{\mathbf{2}}$ & Significación \\
\hline Dependiente, independiente y moderadoras & 648.791 & 0.000 \\
\hline
\end{tabular}

Fuente: elaboración propia.

En la línea de exigencia que tratamos, los coeficientes no estandarizados de la ecuación de la regresión (tabla 6) permiten construir la ecuación de mínimos cuadrados:

Tabla 6. La ecuación de regresión

\begin{tabular}{|l|c|}
\hline \multirow{2}{*}{\multicolumn{1}{c|}{ Modelo }} & Coeficiente no estandarizado \\
\cline { 2 - 2 } & $\mathrm{B}$ \\
\hline Constante & -0.921 \\
\hline Ventas netas & 1.129 \\
\hline Costo de ventas & -1.194 \\
\hline Gastos operativos & -1.045 \\
\hline Otros ingresos/gastos & -1.092 \\
\hline
\end{tabular}

Fuente: elaboración propia. 
Los datos precedentes sugieren que, si las otras variables se mantienen constantes: (a) cada unidad de variación de las ventas netas es seguida de una variación de 1.129 de la variable dependiente; (b) cada unidad de variación del costo de ventas genera una variación de - 1.194 en la variable resultado contable antes de impuestos; (c) cada unidad de variación en los gastos operativos produce una variación de - 1.045 en el resultado contable antes de impuestos; $y$, cada unidad de variación en otros ingresos/gastos causa un cambio de -1.092 en la variable dependiente.

Cuatro son las exigencias relacionadas con la validez del modelo propuesto. La estandarización de los errores permite cumplir con tres de ellas: (a) la relación lineal entre las variables, (b) la presencia (o ausencia) de homocedasticidad, y (c) la independencia de los errores.

Tabla 7. Estadística de residuos

\begin{tabular}{|c|c|c|c|c|c|}
\hline \multicolumn{1}{|c|}{ Variables } & Mínimo & Máximo & Media & $\begin{array}{c}\text { Desviación } \\
\text { estándar }\end{array}$ & $\begin{array}{c}\text { Durbin- } \\
\text { Watson }\end{array}$ \\
\hline $\begin{array}{l}\text { Independiente, } \\
\text { moderadoras y } \\
\text { dependiente }\end{array}$ & -1.523 & 1.176 & 0.000 & 0.745 & 2.897 \\
\hline
\end{tabular}

Fuente: Elaboración propia

Estando a que los valores de los residuos se ubican entre los parámetros -2 (mínimo) y +2 (máximo), puede asumirse que los errores son independientes. Así lo confirma el coeficiente Durbin-Watson (tabla 7).

Una última exigencia, (d) la normalidad en la distribución de los errores ha sido verificada con la ayuda de un histograma. 


\section{Interpretación de los resultados}

La hipótesis alternativa de este estudio propone que el fraude contable es una práctica habitual en nuestro país. Los resultados parecen confirman dicha presunción. Bajo tales premisas, estos serán discutidos e interpretados.

\section{Relación entre las variables independiente y dependiente}

El coeficiente de correlación (0.920) muestra una fuerte relación positiva entre las variables ventas netas y resultados antes de impuestos. Dicha relación, sin embargo, no se manifiesta en la existencia de una variación (crecimiento/decrecimiento) como la que esperábamos: que cada cambio en la variable independiente fuera, seguida por una variación "marginal" (crecimiento/decrecimiento) de la variable dependiente. El comportamiento de ambas expone, más bien, un crecimiento constante de la variable independiente que contrasta con un trazo "flotante" de la variable dependiente.

Figura 2. Relación entre variables vN-RCAI.

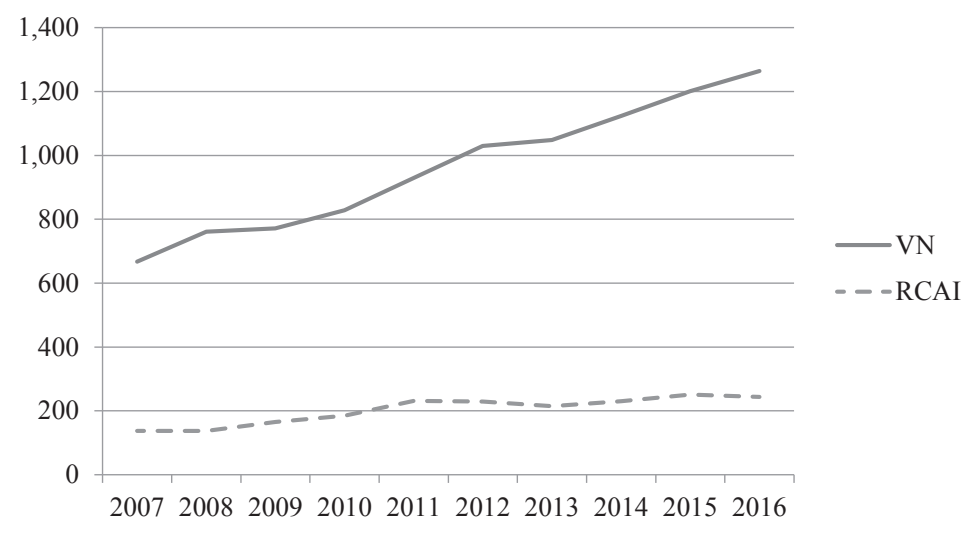

Fuente: elaboración propia.

La cuasiestacionalidad de la variable dependiente se explica por la proporción anual de los valores de dicha variable con respecto a los valores de la 
variable independiente: durante los periodos examinados, los valores de la primera tienden a significar, en promedio, alrededor del $21 \%$ de los valores de la segunda; tal contingencia (a) propone que, por ejemplo, por cada $\mathrm{S} /$ 100.00 de ventas netas se obtiene $S / 21.00$ de ganancias antes de impuestos, y (b) sugiere la existencia de un comportamiento no aleatorio de las cifras (tabla 8).

Tabla 8. Relación porcentual entre variables

\begin{tabular}{|c|c|c|c|c|c|c|c|c|c|c|}
\cline { 2 - 10 } \multicolumn{1}{c|}{} & $\mathbf{2 0 0 7}$ & $\mathbf{2 0 0 8}$ & $\mathbf{2 0 0 9}$ & $\mathbf{2 0 1 0}$ & $\mathbf{2 0 1 1}$ & $\mathbf{2 0 1 2}$ & $\mathbf{2 0 1 3}$ & $\mathbf{2 0 1 4}$ & $\mathbf{2 0 1 5}$ & $\mathbf{2 0 1 6}$ \\
\hline $\mathrm{VN}$ & $100 \%$ & $100 \%$ & $100 \%$ & $100 \%$ & $100 \%$ & $100 \%$ & $100 \%$ & $100 \%$ & $100 \%$ & $100 \%$ \\
\hline RCAI & $21 \%$ & $18 \%$ & $21 \%$ & $22 \%$ & $25 \%$ & $22 \%$ & $20 \%$ & $20 \%$ & $21 \%$ & $19 \%$ \\
\hline
\end{tabular}

Fuente: elaboración propia.

\section{Relación entre las variables ventas netas y costo de ventas}

Como ya se ha dicho, la variable costo de ventas modera la relación entre las variables independiente y dependiente. Así, en la medida en que los valores de costo de ventas se acercan a los valores de ventas netas, dicha eventualidad minimiza la variabilidad de la variable dependiente. En este caso específico, de acuerdo con la figura 3, la premisa parece confirmarse.

Figura 3. Relación entre variables vN-cv.

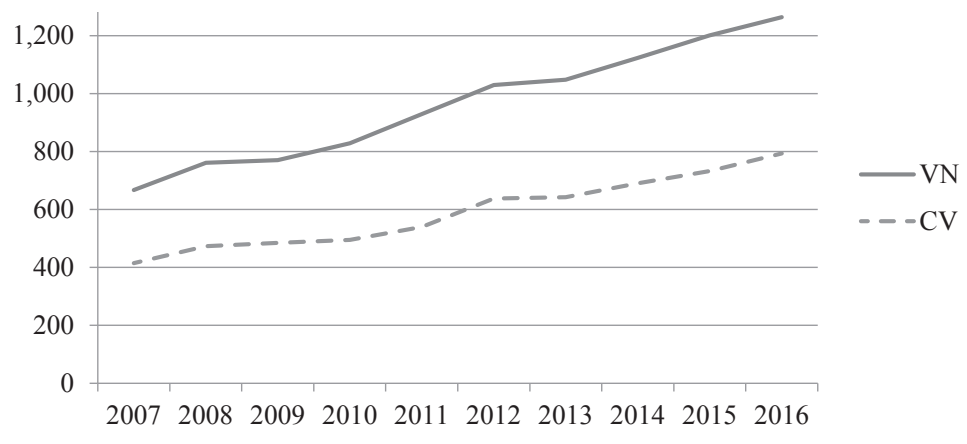

Fuente: elaboración propia. 
Dicho efecto se capta mejor comparando el crecimiento de ambas variables (sobre bases de $100 \%$ para el 2007), a excepción de los periodos 2010 y 2011 las variables aludidas parecen crecer en el tiempo con cierta equidad (tabla 9).

Tabla 9. Crecimiento porcentual anual de las variables.

\begin{tabular}{|c|c|c|c|c|c|c|c|c|c|c|}
\cline { 2 - 10 } \multicolumn{1}{c|}{} & $\mathbf{2 0 0 7}$ & $\mathbf{2 0 0 8}$ & $\mathbf{2 0 0 9}$ & $\mathbf{2 0 1 0}$ & $\mathbf{2 0 1 1}$ & $\mathbf{2 0 1 2}$ & $\mathbf{2 0 1 3}$ & $\mathbf{2 0 1 4}$ & $\mathbf{2 0 1 5}$ & $\mathbf{2 0 1 6}$ \\
\hline $\mathrm{VN}$ & $100 \%$ & $114 \%$ & $116 \%$ & $124 \%$ & $139 \%$ & $154 \%$ & $157 \%$ & $168 \%$ & $180 \%$ & $189 \%$ \\
\hline $\mathrm{CV}$ & $100 \%$ & $114 \%$ & $117 \%$ & $119 \%$ & $130 \%$ & $154 \%$ & $155 \%$ & $166 \%$ & $177 \%$ & $191 \%$ \\
\hline
\end{tabular}

Fuente: elaboración propia.

La figura siguiente soporta la afirmación anterior: en la medida en que los valores de las variables son afines, los trazos (a) se interfieren en algunos periodos, (b) se demarcan en otros y (c) se confunden en los restantes.

Figura 4. Crecimiento de las variables vN-Horizontal-Cv-Horizontal.

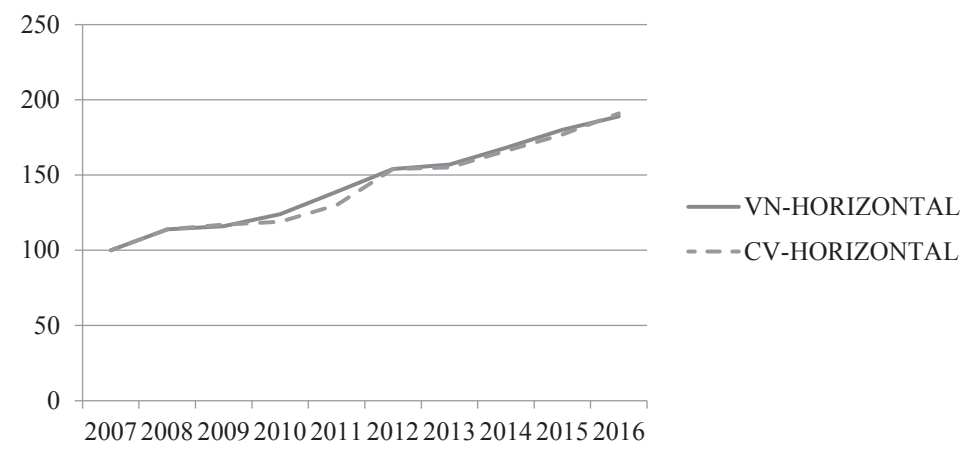

Fuente: elaboración propia.

Otra información complementaria surge de la estimación de la proporción de los valores de costo de ventas con relación a los valores de la variable ventas netas: durante el periodo examinado, la primera representa, en promedio, el $61 \%$ de los valores de la segunda. Ello supone que cada 
S/ 100 de ventas netas acarrea un costo de ventas de S/ 61. Esta contingencia no parece tener la característica de aleatoriedad (tabla 10).

Tabla 10. Proporción de valores de costo de ventas-ventas netas.

\begin{tabular}{|c|c|c|c|c|c|c|c|c|c|c|}
\cline { 2 - 10 } \multicolumn{1}{c|}{} & $\mathbf{2 0 0 7}$ & $\mathbf{2 0 0 8}$ & $\mathbf{2 0 0 9}$ & $\mathbf{2 0 1 0}$ & $\mathbf{2 0 1 1}$ & $\mathbf{2 0 1 2}$ & $\mathbf{2 0 1 3}$ & $\mathbf{2 0 1 4}$ & $\mathbf{2 0 1 5}$ & $\mathbf{2 0 1 6}$ \\
\hline $\mathrm{VN}$ & $100 \%$ & $100 \%$ & $100 \%$ & $100 \%$ & $100 \%$ & $100 \%$ & $100 \%$ & $100 \%$ & $100 \%$ & $100 \%$ \\
\hline $\mathrm{CV}$ & $62 \%$ & $62 \%$ & $63 \%$ & $60 \%$ & $58 \%$ & $62 \%$ & $61 \%$ & $61 \%$ & $61 \%$ & $63 \%$ \\
\hline
\end{tabular}

Fuente: elaboración propia.

\section{Relación entre las variables ventas netas y gastos operativos}

Los gastos operativos representan una segunda variable moderadora en este estudio. Como se esperaba ${ }^{7}$, la figura siguiente muestra un trazo relativamente estable de la variable durante el periodo examinado. Hasta aquí podría hablarse de una influencia no cuestionable en la determinación de la variable dependiente.

Figura 5. Relación entre variables vN-Go.

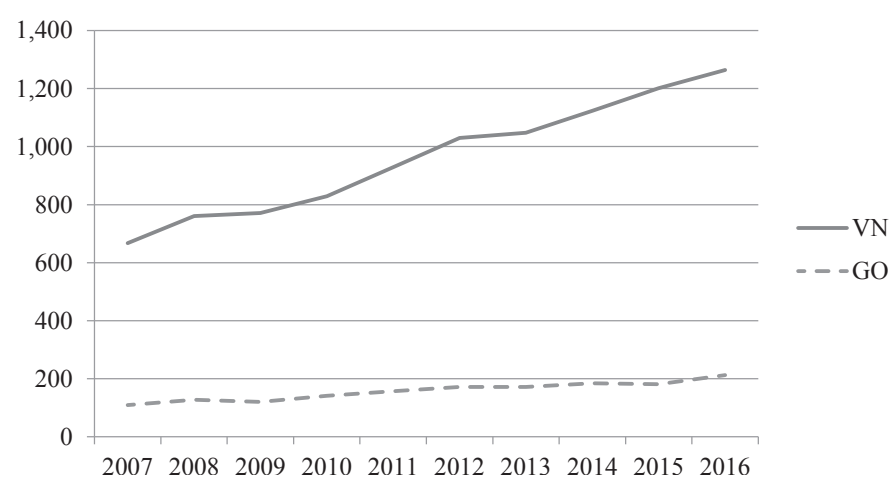

Fuente: elaboración propia.

7 En general, los gastos operativos son aquellos necesarios para ofrecer y poner a disposición de los interesados los productos o servicios que ofrece una empresa. Esta incurre en ellos, aun cuando no venda. 
La estabilidad arriba referida parece explicarse en la proporción de los gastos operativos con respecto a los valores de la variable independiente: durante el periodo examinado los gatos operativos representan, en promedio, el $16 \%$ de los valores de la variable ventas netas. Dicho promedio llama la atención: no parece producto del azar (tabla 11).

Tabla 11. Proporción de valores de gastos operativos-ventas netas.

\begin{tabular}{|c|c|c|c|c|c|c|c|c|c|c|}
\cline { 2 - 10 } \multicolumn{1}{c|}{} & $\mathbf{2 0 0 7}$ & $\mathbf{2 0 0 8}$ & $\mathbf{2 0 0 9}$ & $\mathbf{2 0 1 0}$ & $\mathbf{2 0 1 1}$ & $\mathbf{2 0 1 2}$ & $\mathbf{2 0 1 3}$ & $\mathbf{2 0 1 4}$ & $\mathbf{2 0 1 5}$ & $\mathbf{2 0 1 6}$ \\
\hline $\mathrm{VN}$ & $100 \%$ & $100 \%$ & $100 \%$ & $100 \%$ & $100 \%$ & $100 \%$ & $100 \%$ & $100 \%$ & $100 \%$ & $100 \%$ \\
\hline $\mathrm{CV}$ & $16 \%$ & $17 \%$ & $16 \%$ & $17 \%$ & $17 \%$ & $17 \%$ & $16 \%$ & $16 \%$ & $15 \%$ & $17 \%$ \\
\hline
\end{tabular}

Fuente: elaboración propia.

El análisis del crecimiento horizontal de las cifras, de otro lado, parece mostrar argumentos para una mejor interpretación: en términos porcentuales, en algunos periodos, los gastos operativos crecen en mayor proporción que las ventas netas (p. ej., 2009, 2015). En otras palabras: los primeros crecen conforme crecen las últimas.

Tabla 12. Crecimiento porcentual de las variables.

\begin{tabular}{|c|c|c|c|c|c|c|c|c|c|c|}
\cline { 2 - 10 } \multicolumn{1}{c|}{} & $\mathbf{2 0 0 7}$ & $\mathbf{2 0 0 8}$ & $\mathbf{2 0 0 9}$ & $\mathbf{2 0 1 0}$ & $\mathbf{2 0 1 1}$ & $\mathbf{2 0 1 2}$ & $\mathbf{2 0 1 3}$ & $\mathbf{2 0 1 4}$ & $\mathbf{2 0 1 5}$ & $\mathbf{2 0 1 6}$ \\
\hline $\mathrm{VN}$ & $100 \%$ & $117 \%$ & $110 \%$ & $130 \%$ & $144 \%$ & $157 \%$ & $157 \%$ & $169 \%$ & $166 \%$ & $195 \%$ \\
\hline $\mathrm{CV}$ & $100 \%$ & $114 \%$ & $116 \%$ & $124 \%$ & $139 \%$ & $154 \%$ & $157 \%$ & $168 \%$ & $180 \%$ & $189 \%$ \\
\hline
\end{tabular}

Fuente: elaboración propia.

La figura 6 confirma lo expuesto: en ciertos periodos, los trazos de ambas variables (a) se superponen, (b) se demarcan y (c) se confunden; estas son características de una variable susceptible de ser llamada "instrumental". 
Figura 6. Crecimiento de las variables Go-Horizontal-vN-Horizontal.

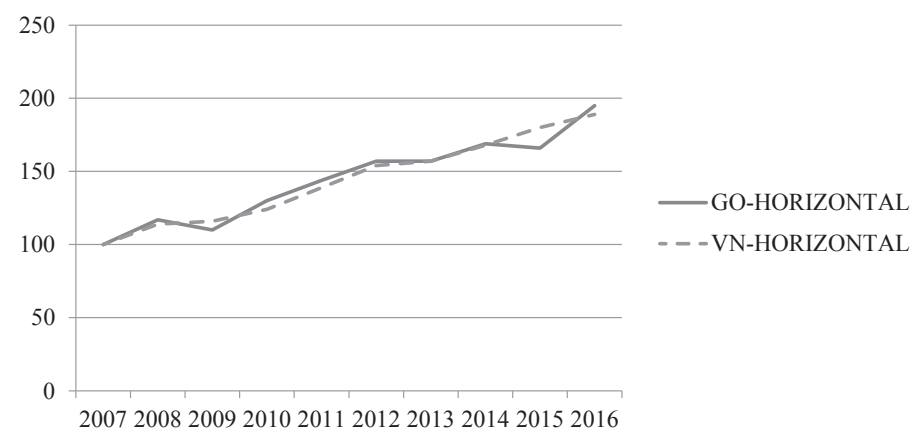

Fuente: elaboración propia.

\section{Relación entre las variables ventas netas y otros ingresos}

Una última variable moderadora también afecta la relación entre las variables independiente y dependiente. Durante el periodo examinado, el trazo de variable moderadora parece mostrar un comportamiento estable. Incluso, si se observa con detenimiento, la línea que representa la trayectoria de la variable aparece completamente plana. Esta característica indicaría que su influencia en la relación, de existir, sería mínima.

Figura 7. Relación entre variables vN-OT.

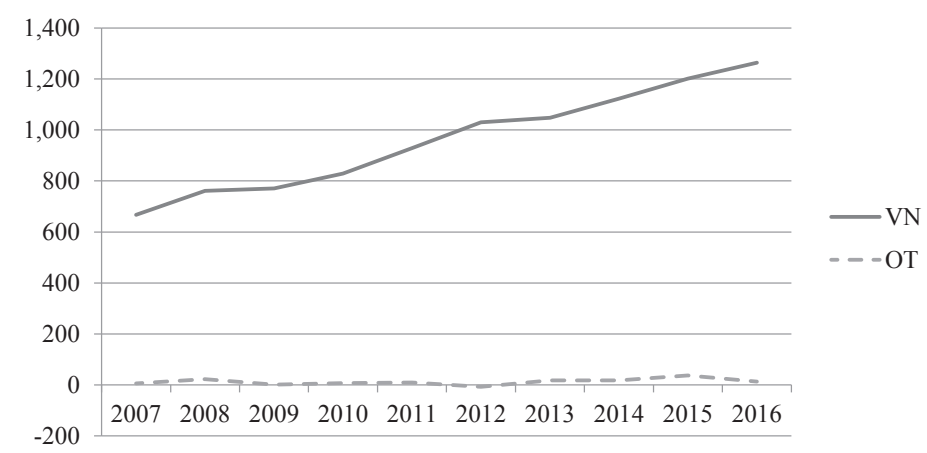

Fuente: elaboración propia. 
El análisis horizontal de las cifras, sin embargo, permite observar una situación diferente: importantes fluctuaciones en los valores asignados a la variable otros ingresos/gastos superan largamente el crecimiento de la variable independiente. Así se observa, en diversos ejercicios anuales (tabla 13).

Tabla 13. Crecimiento porcentual anual de las variables.

\begin{tabular}{|c|c|c|c|c|c|c|c|c|c|c|}
\cline { 2 - 10 } \multicolumn{1}{c|}{} & $\mathbf{2 0 0 7}$ & $\mathbf{2 0 0 8}$ & $\mathbf{2 0 0 9}$ & $\mathbf{2 0 1 0}$ & $\mathbf{2 0 1 1}$ & $\mathbf{2 0 1 2}$ & $\mathbf{2 0 1 3}$ & $\mathbf{2 0 1 4}$ & $\mathbf{2 0 1 5}$ & $\mathbf{2 0 1 6}$ \\
\hline $\mathrm{VN}$ & $100 \%$ & $114 \%$ & $116 \%$ & $124 \%$ & $139 \%$ & $154 \%$ & $157 \%$ & $168 \%$ & $180 \%$ & $189 \%$ \\
\hline $\mathrm{CV}$ & $100 \%$ & $421 \%$ & $17 \%$ & $142 \%$ & $188 \%$ & $-144 \%$ & $348 \%$ & $329 \%$ & $710 \%$ & $239 \%$ \\
\hline
\end{tabular}

Fuente: elaboración propia.

De otro lado, las variaciones proporcionales anuales de la variable moderadora, en comparación con la variable independiente (tabla 14), proponen el carácter instrumental de la variable. En otras palabras, el comportamiento zigzagueante de la variable moderadora no parece provenir de hechos casuales.

Tabla 14. Proporción de los valores otros ingresos/gastos-ventas netas.

\begin{tabular}{|c|c|c|c|c|c|c|c|c|c|c|}
\cline { 2 - 10 } \multicolumn{1}{c|}{} & $\mathbf{2 0 0 7}$ & $\mathbf{2 0 0 8}$ & $\mathbf{2 0 0 9}$ & $\mathbf{2 0 1 0}$ & $\mathbf{2 0 1 1}$ & $\mathbf{2 0 1 2}$ & $\mathbf{2 0 1 3}$ & $\mathbf{2 0 1 4}$ & $\mathbf{2 0 1 5}$ & $\mathbf{2 0 1 6}$ \\
\hline $\mathrm{VN}$ & $100 \%$ & $100 \%$ & $100 \%$ & $100 \%$ & $100 \%$ & $100 \%$ & $100 \%$ & $100 \%$ & $100 \%$ & $100 \%$ \\
\hline $\mathrm{CV}$ & $1 \%$ & $3 \%$ & $0 \%$ & $1 \%$ & $1 \%$ & $-1 \%$ & $2 \%$ & $2 \%$ & $3 \%$ & $1 \%$ \\
\hline
\end{tabular}

Fuente: elaboración propia.

Finalmente, la figura 8 expone una visión más objetiva del comportamiento de la variable otros ingresos/gastos. 
Figura 8. Comportamiento de la variable

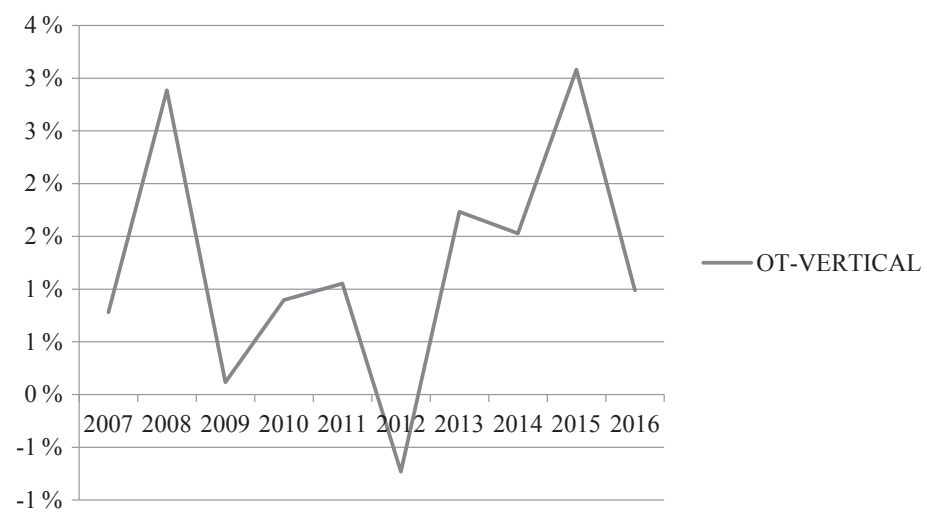

Fuente: elaboración propia.

\section{Discusión de resultados}

Los resultados de nuestro estudio son discutidos e interpretados desde una óptica general. Estos confirman que el fraude contable en la modalidad de estados financieros es una práctica generalizada en nuestro país. Bajo tales premisas, ellos revelan que el método preferido es el de nivelación o lisaje de beneficios. Dichos resultados son coherentes con los estudios desarrollados por Gabás y Pina (1991), Poveda e Iñiguez (2001), Ronen y Sada (1976), quienes proporcionan evidencia de nivelación de resultados. En la misma línea, en un estudio con una muestra canadiense, Breton y Chenail (1997) señalaron, igualmente, que el alisamiento era una práctica muy difundida en ese país. Otra investigación desarrollada por Apellániz (1991) demostró que empresas españolas se abocaban a la práctica del alisamiento de resultados de manera sistemática. Para Cormier et ál. (1998, p. 26), dicha conducta reposa en la hipótesis de la selección contable y tiene por objetivo minimizar la volatilidad de los resultados.

Los resultados alcanzados son sostenidos, igualmente, por supuestos de los modelos teóricos propuestos. Así, en primer lugar, "la oportunidad" 
de cometer un fraude como el estudiado se presenta con la posibilidad de producir información contable.

En dicho escenario, el productor de la información toma ventaja de la asimetría entre emisor/receptor, prerrogativa que se manifiesta mediante la posibilidad de seleccionar la información a revelar y de sesgar la información en función a intereses del emisor. En segundo lugar, "las motivaciones" se sostienen en la hipótesis de maximización: las personas toman decisiones buscando maximizar sus propios beneficios. En el presente caso, los estímulos se manifiestan en los planos (a) económico, p. ej., en la búsqueda de minimizar el pago de impuestos; (b) informativo, p. ej., en la posibilidad de orientar la percepción de los usuarios de la información, y (c) indicativos, p. ej. en la eventualidad de emitir señales a los inversionistas del mercado de acciones.

En tercer lugar, toda conducta fraudulenta es premeditada, es decir “intencional". En la muestra estudiada, la intención es la de engañar (el medio) para orientar la percepción del usuario (el objetivo) de la información (Bayou y Reinstein, 2001). En cuarto lugar, "el esquema" empleado de manera repetitiva ha consistido en establecer un rango (que tiene como base los valores anuales de las ventas netas $=100 \%$ ) en el que los valores de las otras variables oscilan. Así, el costo de ventas representa, como promedio anual, el $61 \%$ de las ventas netas; los gastos operativos significan, en promedio, el $16 \%$; otros ingresos y gastos constituyen el $1 \%$; y los resultados contables antes de impuestos representan, también en promedio, el $21 \%$ de dicha variable.

En quinto lugar, "la acción" (o "la tarea") alude a la actividad necesaria para alcanzar el objetivo; en este caso, seleccionar métodos, procedimientos y técnicas para (a) manipular cifras, (b) tergiversar transacciones, o (c) aplicar "erróneamente" las normas contables. Finalmente, "el objetivo" de las empresas examinadas en este estudio ha sido "reducir la variabilidad de los resultados, que, a la luz de las evidencias, ha sido alcanzado. 
Desde la óptica de la racionalidad instrumental (una teoría sobre la conducta intencionada) conviene recordar los objetivos básicos de la contabilidad: el cálculo de los beneficios y la utilidad de la información. Partiendo de que lo racional es lo útil, la preocupación principal está en la elección de los medios adecuados para alcanzar los fines. En ese contexto, la contabilidad resulta un medio susceptible de dosificación para alcanzar metas predeterminadas (Giraldo, 2007). Este parece haber sido el rol que ha desempeñado la contabilidad en la muestra estudiada.

\section{Conclusiones y perspectivas}

Los resultados obtenidos soportan la hipótesis alternativa propuesta: el fraude contable es una práctica habitual en el Perú. En otras palabras, los estados financieros producidos por las empresas registradas en la Bolsa de Valores de Lima no son veraces, objetivos y confiables. En esa línea, el método preferido por dichas entidades para alcanzar sus fines es el de nivelar 0 alisar los resultados.

Los resultados alcanzados son coherentes con otras investigaciones, los cuales también son sostenidos por teorías como (a) la conducta humana: en un escenario contable es posible pronosticar la conducta cuando se conoce el estímulo, es decir la motivación; (b) la elección racional: en toda sociedad el comportamiento de las personas no es arbitrario; ellas, más bien, desarrollan estrategias orientadas a maximizar sus beneficios; (c) la instrumentalización de la contabilidad: para la racionalidad instrumental, lo racional es lo útil y los objetos son vistos en atención a su función en el logro de un fin específico; (d) la teoría contable positiva: no es posible explicar la práctica contable sin estudiar la racionalidad de los agentes involucrados; y (e) la teoría de la información: en la producción de información financiera, las empresas sesgan información, buscan orientar la percepción de los usuarios, manipulan contenidos buscando distorsionar juicios sobre performances y perspectivas. 
El presente trabajo abre diversas alternativas futuras. Entre ellas, el estudio del fraude (a) mediante la inclusión de sectores empresariales que no formaron parte de la muestra; (b) desde la perspectiva de la calidad de la información que suministran los estados financieros; (c) la confiabilidad de los estados financieros auditados; (d) los efectos de la nivelación de resultados en, por ejemplo, la tributación sobre la renta, la participación de los trabajadores, el pago de dividendos; (e) la contabilidad como instrumento de producción de información, y (f) el cumplimiento de la regulación contable dispuesta por la Superintendencia del Mercado de Valores.

\section{Referências}

Abdullahi R. y Mansor, N. (2015). Fraud triangle theory and fraud diamond theory. Understanding the convergent and divergent for future research. International Journal of Acadmic Research in Accounting Finance and Management Sciences, 5(4), 38-45.

Ajekwe, C. C. M. y Ibiamke, A. (2017). Accounting Fraud: a review of literature. IOSR Journal of Humanities and Social Science (IOSR-JHSS), 22(4), 38-47.

American Institute of Certified Public Accountants. (2002). Auditing Standards Board. Consideration of Fraud in a Financial Statement Audit. Statement on Auditing Standard 99.

Apellániz, G. P. (1991). Una aproximación empírica al alisamiento de beneficios en la banca española. Revista Española de Financiación y Contabilidad, 11(66), 195-219.

Association of Certified Fraud Examiners. (2012). Report to the Nation on Occupational Fraud and Abuse.

Bayou, M. E. y Reinstein, A. (2001). A systemic view of fraud explaining its strategies, anatomy and process. Critical Perspectives on Accounting, 12(4), 383-403.

Breton, G. y Chenail, J-P. (1997). Une étude empirique du lissage des bénéfices dans les entreprises canadiennes. Comptabilité-Contrôle-Audit, 3(1), 53-69.

Carreño, I. (2015, 17 de abril). Dictan cinco años de prisión efectiva para el vicepresidente de la Confiep. La República. Recuperado de https://larepublica. pe/politica/870847-dictan-cinco-anos-de-prision-efectiva-para-el-vicepresidente-de-la-confiep/ 
Cormier, D., Magnan M., y Morard, B. (1998). La gestion stratégique des résultats : le modèle anglo-saxon concient-il au contexte suisse ? Comptabilité-ContrôleAudit, 4(1), 25-48. Dor: https://doi.org/10.3917/cca.041.0025

Carthy, J. D. (1969). La conducta de los animales. Madrid: Biblioteca Salvat.

Chalayer, S. (1995). Le lissage des résultats. Éléments explicatifs avancés dans la littérature. Comptabilité-Contrôle-Audit, 1(2), 89-104.

Feltham, G. A. (1968). The value of the information. The Accounting Review, 43(4), 684-696.

Gabás, T. F. y Pina, M. V. (1991). El alisamiento de beneficios en el sector eléctrico: un estudio empírico. Revista Española de Financiación y Contabilidad, 66, 265-283.

García, B. M. y Humphrey, C. (1995). La auditoria y el fraude: algunas consideraciones internacionales. Revista Española de Financiaclon y Contabilidad, 24(8), 697-725.

Giraldo, G. A. (2007). La cosificación de la contabilidad a través de la racionalidad instrumental de la lógica empresarial. Contaduría Universidad de Antioquia, 50, 133154.

Gonzáles Cruz, M. (2012). Apuntes para una crítica a la teoría de la elección racional a través de la acción comunicativa de Jürgen Habermas. Razón y Palabra, 79. Recuperado de https://www.redalyc.org/articulo.oa?id=199524411052

González, D. J. (2008). Psicología de la motivación. La Habana: Editorial Ciencias Médicas.

Gutiérrez, M. (2017, 26 de junio). Así operaban empresarios y abogados para pagar menos impuestos. Ilusión Fiscal. Recuperado de https://ilusionfiscal. convoca.pe/asi-operaban-empresarios-y-abogados-para-pagar-menosimpuestos.php

Lehman, Ch. R. y Okcabol, F. (2005). Accounting from crime. Critical Perspectives on Accounting, (16), 613-639.

Merkl-Davies, D. M. y Brennan, N. (2007). Discretionary disclosure strategies in corporate narratives: incremental information or impression management? Journal of Accounting Literature, 26, 116-196.

Naranjo, M. L. (2009). Motivaciones: perspectivas teóricas y algunas perspectivas de su importancia en el ámbito educativo. Revista Educación, 33(2), 153-170. 
Odebrecht: dinero para coimas representaba el 0,5 \% al $2 \%$ de la facturación. (2017, 13 de abril). La República. Recuperado de https://larepublica.pe/ politica/865376-odebrecht-dinero-para-coimas-representaba-el-05-al-2-dela-facturacion-de-la-empresa/

Paramio, L. (2000). Decisión racional y acción colectiva. Leviatán, 79, 65-83.

Piaget, J. (2010). La psicología de la independencia. Barcelona: Editorial Crítica.

Poveda, F. e Iñiguez, R. (2001). Alisamiento de beneficios vs. Rentabilidad bursátil: evidencia empírica. Trabajo presentado en el VIII Foro de Finanzas de Madrid 2000. Recuperado de https://dialnet.unirioja.es/servlet/ articulo ?codigo $=2248675$

Proceso. (2005). En WordReference. Recuperado de https://www.wordreference. com/definicion/proceso

Ramamoorti, S. (2008). The psychology and sociology of fraud: integrating the behavioral sciences component into fraud and forensic accounting curricula. Issues in Accounting Education, 23(4), 521-533.

Real Academia Española. (2019). Motivación. En Diccionario de la Lengua Española (edición de tricentenario). Recuperado de https://dle.rae.es/ motivaci\%C3\%B3n?m=form

Robert, M. et ál. (1984). Fondements et étapes de la recherche scientifique en psychologie (2e, éd.). Edisem Inc.

Rodríguez, R. (2014). Economía y sociedad. Esbozo de la sociología comprensiva. Recuperado de http://www.altillo.com/examenes/uba/cbc/sociologia/sociologia_2014_resweber_luch.asp.

Ronen, J. y Sadan, S. (1981). Smoothing income numbers: objective, means and implications. New York: Addison-Wesley.

Se evaden S/ 1.100 mllns en ventas no declaradas. (2018, 6 de diciembre). La República. Recuperado de https://larepublica.pe/economia/1371385-evadens-1100-mllns-ventas-declaradas/

Tremblay D. Cormier, D. y Magnan. M. (1994). Théories et modèles comptables. Développement et perspectives. Quebec, Canadá: Presses de l'Université du Québec.

Vidal de la Rosa, G. (2008). La teoría de la elección racional en ciencias sociales. Sociológica, 23(67), 221-236. 
Villarroya, L. y María, B. (2003). Alteraciones y manipulaciones de la contabilidad [tesis doctoral]. Universidad de Valladolid, España.

Watts, R. y Zimmerman, J. L. (1986). Positive Accounting Theory. Englewood Cliffs , NJ: Prentice-Hall Contemporary Topics in Accounting Series.

Whitley, R. D. (1988). The Possibility and Utility of Positive Accounting Theory. Accounting, Organizations and Society. 13(6), 631-645. 